\title{
Estudio descriptivo de las características sociodemográficas de la discapacidad en América Latina
}

\author{
Descriptive study about social and demographical characteristic \\ of the disability in Latin America
}

Priscila Neves-Silva ${ }^{1}$

Elena Álvarez-Martín ${ }^{1}$
${ }^{1}$ Universidad Rey Juan Carlos. Camino del Molino s/n, Fuenlabrada. 28943 Madrid Espanha. priscila.neves31@gmail.com

\begin{abstract}
The objective of this study was to ascertain the social and demographical characteristics of disability in Latin America and verify the methodology used in the survey by questionnaires for the analysis of disabilities. It is a descriptive study that analyzed the data and methodology of disability surveys conducted in Latin America after 2001. It was noted that there is a big difference in the prevalence of disability found in the surveys that used the International Classification of Functioning, Disability and Health (ICF) for statistical analysis of people with disabilities. It was also shown that in Latin America motor disabilities are more prevalent in men over 65 years old and with a low educational level. The main cause is chronic disease. The conclusion is that ICF is a good methodological tool to use in disability survey questionnaires.
\end{abstract}

Key words ICF, ICIDH, Disabled People, Latin America
Resumen El objetivo de ese estudio es conocer las características socio demográficas de la discapacidad en América Latina y evaluar la metodología utilizada en las encuestas poblacionales para el análisis de la discapacidad. Es un estudio descriptivo que analiza los datos y la metodología de las encuestas de discapacidad de los países de América Latina realizadas después del 2001. Ha sido observado una grande diferencia en la prevalencia de la discapacidad encontrada en las encuestas que utilizaron la CIF para el análisis estadístico de las personas con discapacidad. Además ha sido verificado que en América Latina la discapacidad motora es la más prevalente y acomete mas los hombres con más de 65 años y con bajo nivel educacional. La principal causa es la enfermedad crónica. Concluyese que la CIF es una buena herramienta metodológica para la elaboración de encuestas de discapacidad.

Palabras clave CIF, CIDDM, Personas con discapacidad, América Latina 


\section{Introducción}

La prevalencia de personas con discapacidad en el mundo aumenta cada día más debido, entre otras cosas, al envejecimiento da la población. Los años agregados a la vida aumenta el riesgo de adquirir una discapacidad en las edades avanzadas como secuela de alguna enfermedad o por el deterioro de las capacidades funcionales al envejecer ${ }^{1-6}$.

Sumase al envejecimiento los accidentes de todo tipo, los conflictos armados, la violencia social, el uso abusivo de drogas y alcohol, los desastres naturales, la desnutrición, la marginalización de grupos sociales y la pobreza extrema que son también considerados causas de la deficiencia ${ }^{2-7}$.

La presencia de una deficiencia puede, o no, generar una discapacidad. Antiguamente el modelo médico de la discapacidad veía las personas como incapaces apenas por la presencia de una deficiencia, no en tanto, este concepto ha cambiado y ahora el modelo social de comprensión de la discapacidad percibe que el ambiente tiene un papel importante en este proceso. La relación que una persona que posee una deficiencia establece con el entorno social puede decir sobre su discapacidad.

Las personas con limitaciones físicas, sensoriales o mentales suelen ser incapaces no debido a afecciones diagnosticadas, sino a causa de la exclusión de las oportunidades educativas, laborales y de los servicios públicos ${ }^{1,2,4}$. Esta exclusión se traduce en pobreza y esta pobreza, en lo que constituye un círculo vicioso, aumenta la presencia de deficiencias por cuanto incrementa la vulnerabilidad de las personas ante problemas como la desnutrición, las enfermedades y las condiciones de vida y trabajo poco seguras. Por lo tanto la relación entre pobreza y deficiencia es fuerte y recíproca ${ }^{4,7,8}$.

Para reducir la pobreza y consecuentemente la prevalencia de deficiencias es importante tener un conocimiento tan amplio como sea posible de las características básicas de los diferentes grupos humanos que conforman la sociedad. Este es un primer paso para asegurar una igualdad de oportunidad para todas las personas en todas las esferas de la sociedad. La información estadística coherente, pertinente y oportuna sobre estos grupos, permite que los niveles de decisión puedan establecer un adecuado orden de prioridades para la planificación y ejecución de actividades destinadas a mejorar las condiciones de vida de los mismos, gestionando y administrando con eficiencia los recursos económicos disponibles $^{1-4,9}$.
En ese sentido, un mayor conocimiento de la población con discapacidad, y su relación con variables sociodemográficas y económicas, permite la comprensión global de su situación, y facilita una posterior planificación de las medidas de prevención, asistencia, rehabilitación y creación de servicios en todos los niveles ${ }^{1-3,5,9}$.

Para intentar definir la discapacidad la Organización Mundial de la Salud aprobó en 1976 la CIDDM (Clasificación Internacional de la Deficiencias, Discapacidades y Minusvalías). La CIDDM es un instrumento para la clasificación de las consecuencias de las enfermedades, así como de los traumatismos y otros trastornos, y de sus repercusiones en la vida del individuo. Se basa en el modelo médico y esta esquematizado en tres niveles: deficiencia, discapacidad y minusvalía, pero no muestra suficientemente la relación entre estos conceptos ${ }^{3,5,10}$.

Conceptualmente, el empleo de la CIDDM transformó la manera de considerar las personas con discapacidades. De acuerdo con este marco conceptual, las deficiencias hacen referencia a las anormalidades de la estructura corporal y de la apariencia y a la función de un órgano o sistema, cualquiera que sea su causa; es decir, representan trastornos a nivel de órgano. Las discapacidades reflejan las consecuencias de la deficiencia desde el punto de vista del rendimiento funcional y de la actividad del individuo; por lo tanto, representan trastornos a nivel de la persona. Las minusvalías hacen referencia a las desventajas que experimenta el individuo como consecuencia de las deficiencias y discapacidades; es decir, reflejan la adaptación del individuo al entorno ${ }^{3,5,10}$.

Con respecto al modelo, se criticó su naturaleza unidimensional, el predominio de la perspectiva negativa centrada en las deficiencias, el punto de vista de la discapacidad como hecho individual y el escaso enfoque de los aspectos sociales y contextuales ${ }^{3,5,10-14}$.

Para suplir las deficiencias encontradas en la CIDDM, la OMS aprobó en mayo de 2001 la CIF (Clasificación Internacional del Funcionamiento, de la Discapacidad y de la Salud) La CIF adopta un modelo biopsicosocial, pues reconoce explícitamente que cualquier evaluación del funcionamiento y discapacidad de una persona debe incorporar el contexto físico, cultural y ambiental en el que vive en, $2,11,15-24^{\text {. }}$

Este modelo, por lo tanto, es más adecuado para el entendimiento de las situaciones de discapacidad en diferentes contextos. Su proceso de medida para evaluar la situación de la discapacidad es multidimensional y envuelve funciones, 
estructuras corporales así como las actividades y el contexto ambiental o social de la persona cuya funcionalidad va a ser evaluada. Una vez que describe la funcionalidad, su propósito es valorar los efectos de la enfermedad en la vida cotidiana de los individuos.

La utilización de la CIF como herramienta metodológica en encuestas poblacionales de discapacidad permite la comparación de datos entre países en diferentes momentos a lo largo del tiempo, por lo tanto resulta en una herramienta útil para describir y comparar estado de salud de las poblaciones ${ }^{10-15,17-21,23-25}$. Es decir, la CIF es una herramienta que permite la estandarización de los procesos de obtención de información en un campo prioritario en nuestra sociedad.

Según la Organización Mundial de Salud un $10 \%$ de la población poseen algún tipo de discapacidad y el $80 \%$ vive en países en desarrollo. En América Latina y Caribe esa cifra correspondería a 85 millones de personas. Sumando a estos los amigos, vecinos y familiares que se ven afectados indirectamente por la discapacidad esta cifra sería aún mucho mayor, llegando a más de un cuarto de la población total ${ }^{1,3-5,7,17}$.

No en tanto la obtención de datos de buena calidad para poder estudiar la conexión entre ingreso, pobreza y discapacidad; y para comparar regiones y países es aún muy difícil ${ }^{3,78,25}$. El índice de discapacidad hallado en encuestas de hogar y censos varía drásticamente. Esta variación se debe a las diferentes medidas de discapacidad y diferentes técnicas de recogida de datos, por lo tanto es importante sistematizar y articular la información proveniente de censos y encuestas, así como de los registros administrativos, en un marco conceptual homogéneo y consensuado como es la CIF.

Los objetivos de este estudio son describir las características sociodemográficas de la discapacidad en América Latina y comparar los resultados en función de la herramienta metodológica empleada en las encuestas de las poblaciones.

\section{Material y Métodos}

Se trata de un estudio descriptivo basado en el análisis de datos secundarios de encuestas de discapacidad de América Latina.

Incluyeron en el análisis los siguientes países: Argentina, Brasil, Chile, Colombia, Ecuador, El Salvador, Guatemala, Honduras, Nicaragua, Paraguay, Perú, República Dominicana, Uruguay.
Han sido analizadas las encuestas poblacionales desde el año 2001 al 2009. Se seleccionó el 2001 como inicio del periodo porque se quería analizar las encuestas a partir de la aprobación de la CIF que ha sido en 2001. Se seleccionó la última encuesta de discapacidad existente en cada país en ese periodo.

Se realizó una búsqueda bibliográfica de artículos científicos en Pubmed y Lilacs. Se puso como límite el año de 2001 e idiomas inglés, español y portugués. Las palabras claves empleadas fueron "encuestas Y discapacidad", "discapacidad Y estadística”, “discapacidad Y América Latina”, "prevalencia Y discapacidad", "CIF Y encuestas poblacionales”. La búsqueda se completó consultando diferentes páginas web de organismos internacionales (PAHO, ONU, Banco Interamericano de Desenvolvimiento y Banco Mundial) y de órganos gubernamentales de cada uno de los países analizados como el Instituto Nacional de Estadística de los países.

Para la descripción de la discapacidad en cada país se incluyeron las siguientes variables: edad, sexo, tipo de discapacidad, principal causa de la discapacidad y nivel educacional de las personas con discapacidad. La elección de las variables socio demográficas se basa en el estudio de Osteras et al. ${ }^{21}$ que define que una encuesta poblacional hay que tener en cuenta la edad, el sexo y el nivel educacional de las personas.

\section{Resultados}

De los 13 países analizados 8 poseen encuestas cuya metodología utilizada en su elaboración ha sido la CIF lo que significa $61,5 \%$ de los países, frente a 38,5\% que utilizaron la CIDDM. (Grafico 1). No se ha encontrado el marco teórico utilizado en la encuesta de República Dominicana.

Se observa una gran diferencia en la media de la prevalencia de la discapacidad cuando se utiliza la CIF $(9,6 \%)$ y cuando se utiliza la CIDDM $(2,4 \%)$, que tiene un enfoque en el modelo medico de discapacidad, como herramienta metodológica en las encuestas poblacionales (Grafico 2). Además, la media de prevalencia de la discapacidad cuando se utiliza la CIF se aproxima de la estipulada por la OMS (10\%).

También se observa una diferencia entre la prevalencia total de la discapacidad encontrada en las encuestas de Brasil (14,5\%), Chile (12,9\%), Colombia $(6,4 \%)$, Ecuador $(12,1 \%)$ y Perú $(8,7 \%)$ cuando comparada con los datos de estos 


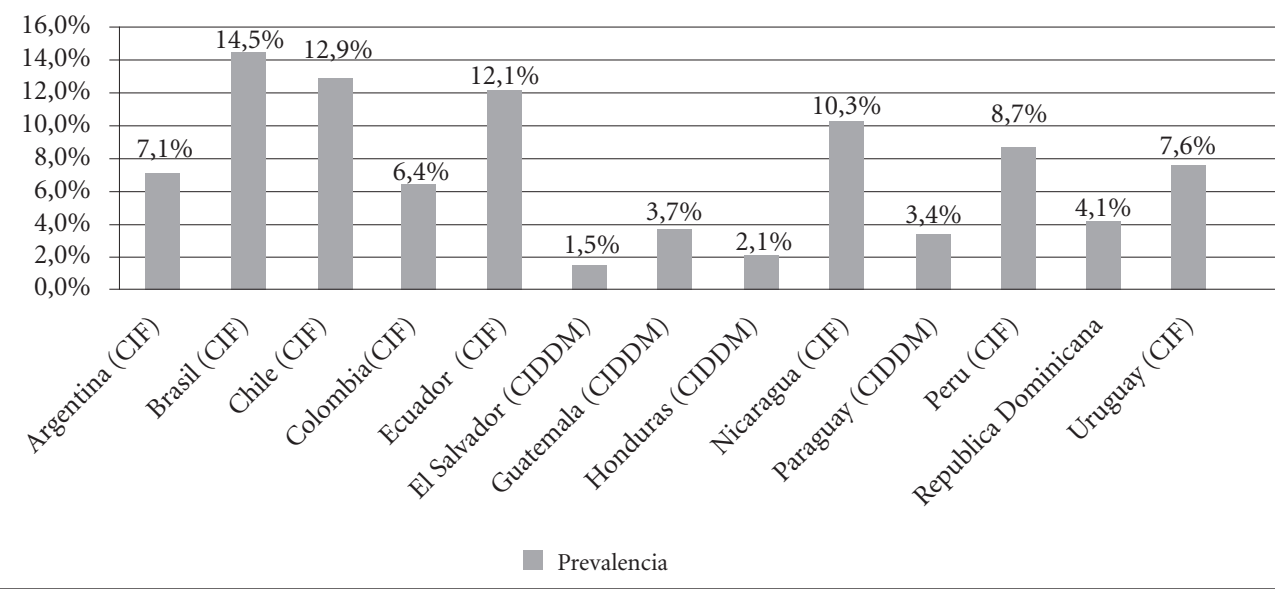

Gráfico 1. Prevalencia de la discapacidad en países de América Latina.

Fuente: Elaboración propia basada en ${ }^{(2,15,21-32)}$.

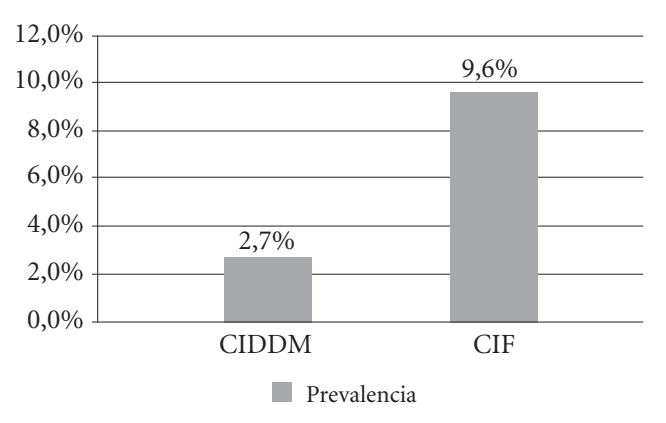

Grafico 2. Relación entre CIDDM X CIF.

Fuente: Elaboración propia basada en ${ }^{(2,15,21-32)}$.

mismos países retirados de encuestas anteriores más que no utilizaron la CIF como marco teórico $(1,4 \% ; 2,2 \% ; 1,9 \% ; 4,7 \%$ y $1,3 \%$ respectivamente) (Grafico 3).

$\mathrm{Al}$ analizar los datos se observa que la discapacidad en América Latina es más prevalente en el área rural en cinco países (Ecuador, El Salvador, Guatemala, Honduras y República Dominicana). En Argentina, Brasil, Chile, Nicaragua y Uruguay la prevalecía es mayor en área urbana ${ }^{2,16,22,23,25-31,33-35}$.

El tipo de discapacidad mas prevalente es la motora en $90,9 \%$ de los países. Solo Brasil y Ecuador poseen la discapacidad visual como más prevalente. En República Dominicana, 20\% de la discapacidad son de tipo sensorial, dentro de este grupo existe una mayor proporción de personas ciegas (Tabla 1 e Grafico 4).

Los hombres son más acometidos que las mujeres en $66,7 \%$ de los países analizados. Argentina, Brasil, Chile, Nicaragua y Uruguay poseen mayor prevalencia de la discapacidad entre las mujeres. En el análisis por edad verificase que $75 \%$ de los países tienen la discapacidad más prevalente en personas mayores de 65 años, solo Chile y Guatemala poseen mayor prevalencia de la discapacidad en personas por debajo de 65 años (Grafico 4).

Las enfermedades crónicas son consideradas como la principal causa de la discapacidad en $63,6 \%$ de los países, en Paraguay son las enfermedades en el periodo perinatal las que generan más discapacidad. En 91\% de los países la discapacidad acomete más a las personas con bajo nivel educacional (Grafico 4).

Sobre el nivel educacional de las personas con discapacidad si observa que en Chile $8,5 \%$ de las personas con discapacidad están estudiando, a diferencia del $27,5 \%$ de la población total del país que estudia actualmente. Además $10 \%$ de la población con discapacidad no cuentan con ningún año de estudio y un $42 \%$ no ha completado la enseñanza básica ${ }^{29}$.

En Colombia casi $40 \%$ de los discapacitados no poseen ningún tipo de escolaridad ${ }^{30}$. En Ecuador $58,8 \%$ solo poseen educación primaria ${ }^{16}$. En El Salvador $44,8 \%$ son analfabetos mientras en la población general el índice de analfabetismo es de $15,9 \%{ }^{16}$. En Guatemala 52\% no poseen nivel de escolaridad $^{33}$, en Honduras 53\% de las personas 


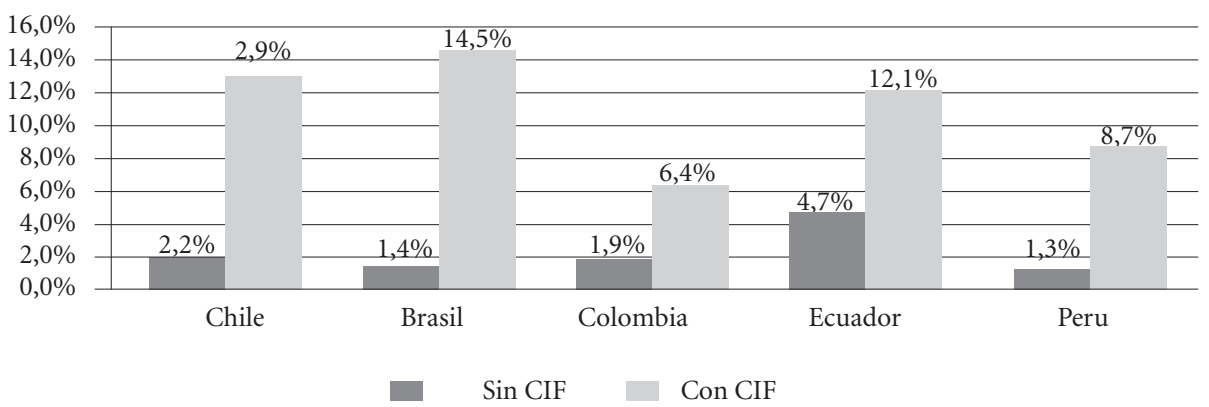

Gráfico 3. Prevalencia de la discapacidad en Países de América latina calculada con y sin la CIF

Fuente: Elaboración propia basada en ${ }^{(2,15,21-32)}$.

Tabla 1. Porcentaje de discapacidad por deficiencias en países de América Latina.

\begin{tabular}{lrrrrrr}
\hline \multicolumn{1}{c}{ Países } & Motora & Visual & Auditiva & Habla & Mental & Otra \\
\hline Argentina & $41,9 \%$ & $19,5 \%$ & $16,5 \%$ & $2,9 \%$ & $16,4 \%$ & $2,8 \%$ \\
Brasil & $22,9 \%$ & $48,1 \%$ & $16,7 \%$ & $\mathrm{NS}$ & $8,3 \%$ & $4,1 \%$ \\
Chile & $31,3 \%$ & $19,0 \%$ & $8,7 \%$ & $\mathrm{NS}$ & $16,8 \%$ & $25,3 \%$ \\
Colombia & $53,8 \%$ & $44,6 \%$ & $24,5 \%$ & $26,5 \%$ & $\mathrm{NS}$ & $6,8 \%$ \\
Ecuador & $52,7 \%$ & $77,9 \%$ & $28,2 \%$ & $\mathrm{NS}$ & $14,7 \%$ & $36,3 \%$ \\
El Salvador & $48,6 \%$ & $26,5 \%$ & $21,1 \%$ & $37,0 \%$ & $36,0 \%$ & $1,0 \%$ \\
Guatemala & $34,0 \%$ & $27,0 \%$ & $18,0 \%$ & $6,0 \%$ & $13,0 \%$ & $1,0 \%$ \\
Honduras & $18,1 \%$ & $6,9 \%$ & $5,2 \%$ & $4,7 \%$ & $3,9 \%$ & $\mathrm{NS}$ \\
Nicaragua & $40,0 \%$ & $10,0 \%$ & $32,0 \%$ & $11,0 \%$ & $\mathrm{NS}$ & $\mathrm{NS}$ \\
Paraguay & $25,6 \%$ & $14,9 \%$ & $0,1 \%$ & $10,9 \%$ & $25,5 \%$ & $\mathrm{NS}$ \\
República Dominicana & $24,0 \%$ & $\mathrm{NS}$ & $\mathrm{NS}$ & $\mathrm{NS}$ & $12,0 \%$ & $\mathrm{NS}$ \\
Uruguay & $36,6 \%$ & $25,0 \%$ & $13,6 \%$ & $2,4 \%$ & $5,4 \%$ & $17,1 \%$
\end{tabular}

Fuente: Elaboración propia basada en $\left({ }^{2,15,21-32}\right)$. Nota: Una persona puede estar en más de una categoría. NS: No se sabe el dato.

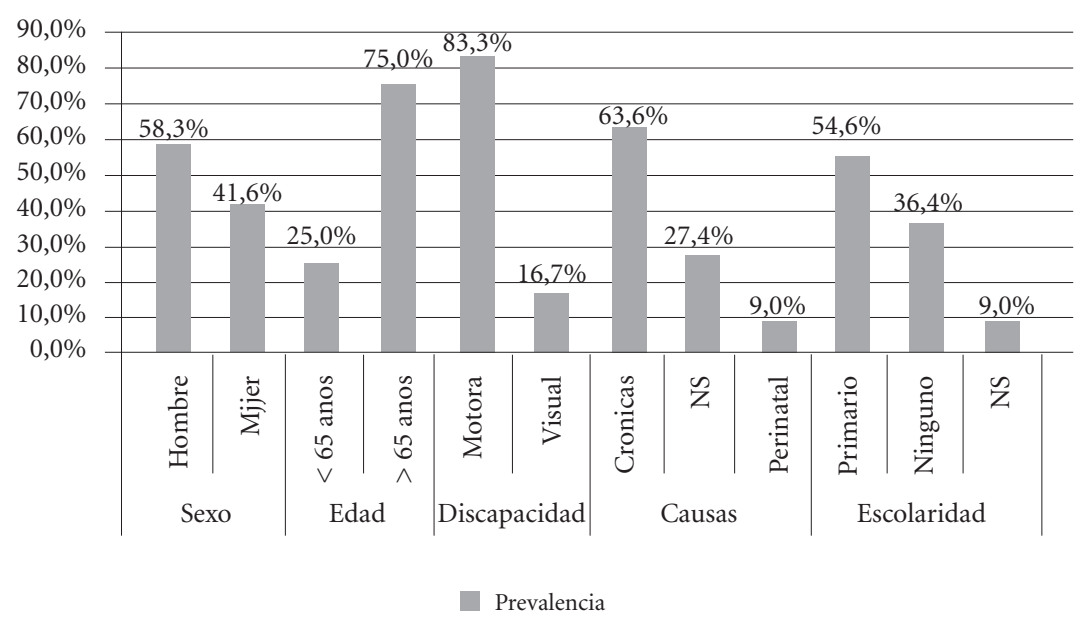

Gráfico 4. Característica de la Discapacidad en América Latina. 
con discapacidad no han alcanzado ningún nivel educativo, mientras que en la población total esa cifra es de $32 \%$; además, $51 \%$ de las personas con discapacidad, mayores de 15 años, no saben leer ni escribir, para la población general, en este mismo rango de edad, este porcentaje es de $20 \%{ }^{34}$. En Nicaragua $45 \%$ no tienen instrucción ${ }^{36,37}$. La Tasa de analfabetismo en la población con discapacidad en Paraguay es de $43 \%$ a diferencia del $7 \%$ que corresponde a la tasa de analfabetismo en la población general sin discapacidad ${ }^{38}$. En República Dominicana 70\% solo tienen educación básica y el 36.5\% no sabe leer ni escribir ${ }^{39}$. En Uruguay el alto porcentaje de personas con discapacidad de 25 años o más de edad que carecen o tienen muy bajo nivel de instrucción (37.7\%), contrasta con el observado para la población sin discapacidad $(12.6 \%)^{2}$.

\section{Discusión}

La definición y la mensuración de la discapacidad es un tema de creciente interés. Los cambios demográficos y el envejecimiento de la población aumentan el riesgo de adquirir algún tipo de discapacidad decurrente de una enfermedad crónica. La ausencia de una definición clara sobre "discapacidad" es un grande impedimento para la promoción de la salud de personas con deficiencia. Por lo tanto, la vigilancia y la intervención dependen de la capacidad para identificar las personas que deberían ser incluidas en esa clasificación ${ }^{6,10,12,15,17,23,25}$.

En el ámbito de la información sanitaria necesaria para la importante misión de conocer el estado de salud de las poblaciones, contamos con herramientas tales como las clasificaciones, los sistemas operativos de recogida de datos y los indicadores sintéticos de salud.

La Clasificación del Funcionamiento, de la Discapacidad y de la Salud (CIF) pertenece a la "familia" de clasificaciones internacionales desarrolladas por la Organización Mundial de la Salud (OMS), que pueden ser aplicadas a varios aspectos de salud. Esta familia proporciona el marco conceptual para clasificar un amplio rango de información relacionada con la salud y emplea un lenguaje estandarizado y unificado, que posibilita la comunicación sobre la salud y la atención entre diferentes disciplinas y ciencias en todo el mundo ${ }^{9,24}$.

Por lo tanto la CIF, es un marco conceptual que permite evaluar la salud de la población. El termino discapacidad se refiere a deficiencias, li- mitaciones en las actividades y restricciones en la participación social; ya el termino funcionalidad incluye funciones, estructuras corporales, actividad y participación ${ }^{9,24}$. Al analizar estos términos se comprende que el concepto de personas con discapacidad no pasa apenas por la presencia de una deficiencia, pero también, en la forma de relación de las personas con el ambiente y la sociedad. Una deficiencia puede generar una discapacidad si la persona no consigue participar de las actividades sociales de manera inclusiva, pero cuando el ambiente se encuentra preparado para recibir las diferencias una persona con deficiencia puede no ser incapaz. El concepto de discapacidad incluye la relación de la persona con el medio en que vive y la estructura social que esta inserida.

La CIF es, también, una importante herramienta en la práctica clínica. A la hora de hacer la evaluación de un paciente la CIF no solo ayuda a describir las condiciones del paciente pero, también, el impacto de los factores socio-ambientales en la funcionalidad del individuo ${ }^{7-9,24}$. Pero la escasa divulgación de esta herramienta en los centros de formación profesional y en los servicios de salud no permite que los profesionales estén familiarizados con la aplicación de la CIF lo que compromete su uso en la clínica. Cuando los profesionales de salud utilizan la CIF en la evaluación de sus pacientes, ellos consiguen tener una visión más ampliada e integral de los problemas que afectan la funcionalidad. Además, una vez que tiene un lenguaje universal, su uso auxilia la comunicación entre los miembros del equipo de salud, y entre los profesionales y el paciente, lo que facilita la toma de decisiones ${ }^{32}$.

Según muchos autores ${ }^{10,23-25}$ la CIF también promueve una estructura muy bien definida a la hora de hacer la encuesta, lo que permite la comparación de cuestiones poblacionales entre países. Para que los dados sean válidos, fiables y comparables es importante que todos los países consigan comprender el real significado de los conceptos de discapacidad y de funcionalidad. Este ha sido un grande dificultador para la recogida de dados antes de la elaboración de la CIF. Según Di Nubila y Buchalla ${ }^{3}$ la CIF es el modelo conceptual de la OMS para la salud e discapacidad y su uso permite la elaboración de políticas públicas para la personas con discapacidad. La definición de las áreas de funcionalidad auxilia los países a entender los datos de las encuestas una vez que facilita la elaboración de las preguntas. Además la CIF genera nuevos conceptos en relación a la capacidad en realizar una actividad 
y la participación social, y las preguntas pasan a tener el potencial para diferenciar ambas cosas. Cuando la CIF es utilizada en las políticas públicas es posible elaborar planos de acción en el área del trabajo, educación, salud, urbanización, asistencia y participación social, que promuevan la inclusión. Países como Alemania, Australia, Estados Unidos, Japón, Irlanda y Portugal tienen ejemplos de buenas prácticas con el uso de la CIF en las políticas públicas ${ }^{32}$.

En nuestro estudio se ha evaluado 13 países, 4 utilizaron la CIDDM (El Salvador, Guatemala, Honduras y Paraguay) y 8 la CIF (Argentina, Brasil, Chile, Colombia, Ecuador, Nicaragua, Perú, Uruguay) como herramienta metodológica en la elaboración de sus encuestas poblacionales, lo que enseña una aceptación de muchos países en el uso de ese modelo de clasificación. La diferencia encontrada entre los datos de prevalencia de las encuestas que utilizaron la CIF y las que utilizaron la CIDDM, u otra, es notable. Se observa que en los países que utilizaron la CIF sus datos de prevalencia se aproximan a lo estipulado por la OMS con una media de $9.6 \%$, mientras que los demás se quedan muy lejos de ese valor (media de 2,7\%). En el estudio de Mbogoni ${ }^{38}$ que evaluó datos de discapacidad en diversos países por medio de la base de datos DISTAT (The United nations Disability statistics Database) observase que todas las encuestas anteriores a la CIF demuestran una prevalencia de la discapacidad en torno de 2,3\%. Por lo tanto se puede concluir que la utilización de la CIF favorece la identificación de la discapacidad en la población. No en tanto, es importante que la CIF sea conocida en otras instancias gubernamentales. El desconocimiento de esta herramienta en otras esferas del gobierno impide el uso correcto de los dados y, consecuentemente, la elaboración de políticas públicas más eficaces para esta población.

Con relación a las características sociodemográficas de la discapacidad este estudio comprueba la relación directa que hay entre envejecimiento, enfermedades crónicas y discapacidad ya señalada por otros autores s-7,28,35 $^{\text {. La hipertensión }}$ arterial, la diabetes y el ictus son enfermedades que muchas veces promueven discapacidad como la ceguera y las discapacidades motoras, que son los tipos de discapacidades más prevalentes en América Latina según este estudio y que coincide con otros ${ }^{4,39}$. Estas enfermedades se relacionan directamente con el envejecimiento, por lo tanto, su prevalencia aumenta a cada día debido al cambio demográfico observado en muchos países. Los hábitos de vida son considerados los principales factores de riegos para estas enfermedades, siendo así, actividades de promoción y protección de la salud son imprescindibles en la lucha contra las discapacidades.

A pesar de observar una grande prevalencia de la discapacidad en edades más avanzadas, no podemos olvidar que uno de los factores de la discapacidad es la precariedad de atención perinatal. El desarrollo es uno de los factores que dificultan el diagnóstico de la discapacidad en niños, pues la experiencia de la discapacidad no es igual a de los adultos ${ }^{2}$. Por lo tanto estos niños aún viven en la invisibilidad, poca atención, por parte de los gobiernos, son dadas a ellos, lo que repercute en la coleta de dados. Para muchos países un niño con discapacidad no podrá tornar-se un adulto que contribuya económicamente para su país, y esto resulta en escasas políticas públicas que favorezcan su inclusión y participación social. Este es un factor que necesita una discusión más amplia entre los legisladores pues el acceso adecuado a los servicios de salud favorecen la detección temprana de la discapacidad, lo que es fundamental para una rehabilitación eficaz y para la inclusión en la escuela y en el trabajo. Para facilitar la comprensión de la discapacidad de los niños las autoras Di Nubila y Buchalla ${ }^{3}$ afirman la necesidad de elaboración de un instrumento que pueda mensurar de modo distinto las condiciones de salud crónicas, las discapacidades y los factores ambientales que inciden en la discapacidad.

Con respecto a la diferencia por sexos este estudio encontró un mayor acometimiento en hombres (Colombia, Ecuador, El Salvador, Guatemala, Honduras, Paraguay, Republica Dominicana) que mujeres, confirmando lo que esta descrito por muchos autores ${ }^{1,4,5,7,12,17,40,41}$.

No en tanto, se percebe que en $57 \%$ de los países que utilizaron la CIF como herramienta metodología (Argentina, Brasil, Chile, Nicaragua y Uruguay) la mujer es mas acometida que el hombre lo que coincide con el estudio de Osteras et al. ${ }^{21}$ que también utilizó la CIF para elaborar una encuesta en Suecia. Una vez que las mujeres viven más que los hombres la población femenina, incluyendo la con discapacidad, tiende a ser mayor. Además en algunas culturas la desigualdad en el tratamiento de los dos sexos es muy grande lo que incrementa la tasa de mortalidad de mujeres con discapacidad frente a los hombres. La violencia contra las mujeres también es otro factor que genera altas tasas de deficiencia en este grupo y esta práctica aún es muy común en países en vías de desarrollo. Es necesaria una investigación más profunda del tema para esta- 
blecer con mayor precisión la relación entre sexo y discapacidad.

En este estudio observase también una relación directa entre la discapacidad y la pobreza ya relatada en estudios anteriores ${ }^{4,5,7,27,28,35}$. En todos los países evaluados la prevalencia de la discapacidad es mayor en las personas con bajo o ningún nivel educacional. Muchos estudios ${ }^{4,7,15,27,28,35,41}$ también relatan que las personas con discapacidad sufren una limitación de oportunidades en el mercado laboral y en el ingreso económico cuando comparado con una persona sin discapacidad, lo que genera una mayor dependencia, por parte de la persona con discapacidad, para su supervivencia, $y$, consecuentemente incrementa la pobreza.

Tornase importante despertar la consciencia de la población en relación a los beneficios resultantes de la inclusión de la personas con discapacidad no solo para los individuos pero también para la sociedad. Las personas que poseen una deficiencia deben ser incluidas en todas las esferas sociales, económicas y políticas.

La participación de la sociedad civil a través de las organizaciones no gubernamentales puede, de muchas maneras, apoyar las acciones gubernamentales para estas personas, sugiriendo soluciones y ofreciendo servicios complementares que auxilien en la inclusión social.

\section{Conclusión}

La discapacidad en países en vías de desarrollo se relaciona con envejecimiento, malnutrición, enfermedades crónicas, baja calidad de asistencia perinatal, accidentes y violencia. Sumase a esto las precarias condiciones de vida que disminuyen la resistencia del individuo - las inadecuadas instalaciones sanitarias aumentan el riesgo a contraer enfermedades infecciosas - y la dificultad en tener acceso a las vacunas.

Este es un obstáculo para el desarrollo de los países. La superación de estos y la creación de condiciones propicias para que las personas con discapacidad aporten al desarrollo económico y social de su país pueden ayudar a reducir los niveles de pobreza de toda la comunidad. A menos que estos individuos sean integrados a las actividades del desarrollo, será muy difícil alcanzar los Objetivos de desarrollo del milenio antes del año 2015.

El informe de discapacidad de las Américas desarrollado por el Banco Interamericano en 2004 relata que $82 \%$ de las personas con discapacidad viven en la pobreza. Ellas son excluidas de manera generalizada de la vida social, económica y política de la comunidad, sea debido a la estigmatización directa o a la falta de consideración de sus necesidades en el diseño de políticas, programas y servicios.

El primer paso para reducir la discriminación y el abandono político y social de las personas con discapacidad y promover la integración y la igualdad es tener una herramienta capaz de colectar datos estadísticos con fiabilidad y conocer la situación social de las personas. Pero este es todavía uno de los grandes problemas encontrados en América Latina.

Hay una grande dificultad en encontrar datos sobre la discapacidad en estos países. Es decir, la elaboración de más estudios de investigación sobre la prevalencia de la discapacidad, sus características socio-demográficas y sus factores de riesgo en América Latina y en todo el mundo es de suma importancia. La falta de información es uno de los mayores problemas en este campo y esta es una herramienta capaz de hacer visible las personas con discapacidad y, consecuentemente, mejorar su calidad de vida.

Es importante incentivar la elaboración de una encuesta única, basada en la CIF, para todos los países a fin de conseguir colectar datos homogéneos. La utilización de la CIF en encuestas poblacionales puede contribuir de una manera muy positiva para el establecimiento de políticas públicas dirigidas a las personas con discapacidad, promover la igualdad de oportunidades, asegurar sus derechos humanos y apoyar la lucha contra la discriminación una vez que auxilia en la elaboración de una herramienta internacional fiable de coleta de datos.

Las personas con discapacidad necesitan hacerse visibles y para ello requieren de información confiable que permita conocer su situación. 


\section{Colaboración}

P Neves-Silva fue responsable por la coleta y el análisis de los datos, y elaboración del artículo. E Álvarez-Martín hizo la revisión final.

\section{Referencias}

1. Fundación Par. La discapacidad en Argentina: un diagnóstico de situación y políticas públicas vigentes a 2005. Buenos Aires: Fundación Par; 2006.

2. República Oriental del Uruguay. Instituto Nacional de Estadística (INE). Comisión Nacional Honoraria del Discapacitado. Encuesta Nacional de Personas con Discapacidad: Informe Final. Montevideo: INE; 2004.

3. Di Nubila H, Buchalla C. O papel das Classificações da OMS - CID e CIF nas definições de deficiência e incapacidade. Rev. bras. epidemiol. 2008; 11(2):324-335.

4. Banco Interamericano de Desarrollo (BID). Encuesta de discapacidades en países de América Latina. Washington: BID; 2006.

5. Van Brakel WH, Officer A. Approches and tools for measuring disability in low and middle-income countries. Lepr Rev 2008; 79(1):50-64.

6. Alves LC, Leite IC, Machado CJ. Conceituando e mensurando a incapacidade functional da população idosa: uma revisão de literatura. Cien Saude Colet 2008; 13(4):1199-1207

7. Stucki G1, Kostanjsek N, Ustün B, Cieza A. ICF- based classification and measurement of functioning. Eur J Phys Rehabil Med 2008; 44(3):315-328.

8. Cieza A, Stucki,G. The international Classification of Function Disability and Health: its development process and content validity. Eur J Phys Rehabil Med 2008; 44(3):303-313.

9. World Health Organization (WHO). International Classification of functioning, disability and health: ICF. Geneve: WHO; 2001.

10. Ustun T, Chatterji S, Bickenbach J, kostanjsek N, Schneider M. The International Classification of Functioning, Disability and Health: a new tool for understanding disability and health. Disabil Rehabil 2003; 25(1112):565-571.

11. Perrin J, Hicks P. The Future of Disabilities in America: review of institute of medicine report. Ambulatory Pediatrics 2008; 2:71-72.

12. Jelsma J. Use of the international classification of functioning, disability and health: a literature survey. $J$ Rehabil Med 2009; 41(1):1-12.

13. Swanson G, Carrothers L, Mulhors K. Comparing disability survey questions in five countries: a study using ICF to guide comparisons. Disabil Rehabil 2003; 25(1112):665-675.

14. Organización Panamericana de la Salud. Discapacidad: lo que todos debemos saber. Rev. Inst. Med. trop. 2006; 48(3):146-146.

15. Viveros A, Ríos M. América Latina: Banco Mundial pide la adopción de medidas sobre la discapacidad y la pobreza, como se celebra el Día Internacional de las Personas con Discapacidad. Washington: Banco Mundial; 2006.

16. Guatemala. Agencia Española de Cooperación Internacional (AECID), Real Patronato sobre Discapacidad, Ministerio de Trabajo y Asuntos Sociales, Fundación ACS. Las Personas con Discapacidad en Centro América. Ciudad de Guatemala: AECID; 2007.

17. Montes A, Massiah E. Disability Data: Survey and Methods Issues in Latin America and the Caribbean. Washington: Inter-American Development Bank (IDB); 2004. 
18. Urquieta-Salomón JE, Figueroa JL, Hernández-Prado B. El gasto en salud relacionado con la condición de discapacidad. Un análisis en población pobre de México. Salud Publica Mex 2008; 50(2):136-146.

19. Dudzik P, Elwan A, Metts R. Disabilities policies, statistics, and strategies in Latin America and the Caribbean: a review. Washington: Inter-American Development Bank (IDB); 2006.

20. Hernandez-Jaramillo J, Hernandez-Umaña I. Una aproximación a los costos indirectos de la discapacidad en Colombia. Rev Salud Publica (Bogota) 2005; 7(2):130-140.

21. Osteras N, Brage S, Garrant A, Benth J, Natvig B, Gulbrandsen P. Functional Ability in a population: normative survey data and reliability for the ICF based Norwegian Function Assessment Scale. BMC Public Health 2007; 7:278.

22. Madden R, Choi C, Sykes C. The ICF as a framework for national data:the introduction of ICF into Australian data dictionaries. Disabil Rehabil 2003; 25(1112):676-682.

23. Roussel P, Barral C. Reference to ICIDH in French surveys on disability. Disabil Rehabil 2003; 25(11-12):659664.

24. Ferreira LTD, Castro SS, Buchalla CM. The International Classification of Functioning, Disability and Health: progress and opportunities. Cien Saude Colet 2014; 19(2):469-474.

25. Buñuales M, Diego P, Moreno J. La Classificacion Internacional del Funcionamento de la discapacidad y de la salud (CIF) 2001. Rev Esp Salud Publica 2002; 76(4):271-279.

26. Costa Rica. Instituto Nacional de Estadísticas y Censos (INEC). Censo poblacional. San José: INEC; 2001.

27. Almada C. Discapacidad en el Paraguay. Assunción: Banco Interamericano de Desarrollo (BID); 2002.

28. Argentina. Instituto Nacional de Estatisticas y Censos (INDEC). Encuesta Nacional de personas con discapacidad. Buenos Aires: INDEC; 2003.

29. Chile. Instituto Nacional de Estadística (INE). Primer estudio nacional de la discapacidad e informes regionales 2004. Santiago de Chile: INE; 2004

30. Colômbia. Departamento Administrativo Regional de Estadísticas (DANE). Censo general 2005 Discapacidad Personas con limitaciones permanentes. Bogotá: DANE; 2006.
31. Obando-Ante LM, Lasso-Benavides AE, Vernaza-Pinzon P. Discapacidad en población desplazada en el Cauca, Colombia. Rev Salud Publica (Bogota) 2006; 8(2):182-190.

32. Fontes AP, Fernandes AA, Botelho MA. Funcionalidade e incapacidade: aspectos conceptuais, estruturais e de aplicação da Classificação Internacional de Funcionalidade, Incapacidade e Saúde (CIF). Rev. Port. Sau. Pub. 2010; 28(2):171-178.

33. Guatemala. Instituto Nacional de Estadística (INE). Encuesta Nacional de Discapacidad. Ciudad de Guatemala: INE; 2005.

34. Honduras. Instituto Nacional de Estadística (INE). La Discapacidad en Honduras. Análisis de los Resultados de la XXVI Encuesta Permanente de Hogares de Propósitos Múltiples. Tegucigalpa: INE; 2002

35. Perú. Instituto Nacional de Estadística (INE). Perfil sociodemografico del Perú. Census Nacionales 2007. Lima: INE; 2007.

36. Nicaragua. Instituto Nacional de Estadísticas y Censos (INEC). Encuesta nicaragüense para personas con discapacidad. Manágua: INEC; 2003.

37. Pizzolitto G. Informe sobre Personas con Discapacidad en Nicaragua. Manágua: IBD; 2004.

38. Mbogoni M. On the application of the ICIDH and ICF in developing countries: evidence from the United Nations Disabilities Statistics Database (DISTAT). Disabil Rehabil 2003; 25(11-12):644-658.

39. República Dominicana. Comisión Nacional de la Sociedad de la Información y el Conocimiento. Cifras sobre las personas con discapacidad en Republica Dominicana. Santo Domingo: Indotel; 2008.

40. Brasil. Ministério da Justiça (MJ). Relatório sobre a prevalência de deficiências, incapacidades e desvantagens. Rio de Janeiro: MJ; 2004.

41. Rosso V. Bolivia: Caracteristicas Sociodemograficas de la Población con Discapacidad. Santiago de Chile: Banco Interamericano de Desarrollo; 2004.

Artigo apresentado em 16/09/2013

Aprovado em 13/05/2014

Versão final apresentada em 17/05/2014 